\title{
Right heart failure: toward a common language
}

\author{
Mandeep R. Mehra, ${ }^{1}$ Myung H. Park, ${ }^{2}$ Michael J. Landzberg, ${ }^{1}$ Anuradha Lala, ${ }^{1}$ \\ Aaron B. Waxman,' on behalf of the International Right Heart Failure \\ Foundation Scientific Working Group
}

'Brigham and Women's Hospital Heart and Vascular Center and Harvard Medical School, Boston, Massachusetts, USA; ${ }^{2}$ Department of Medicine (Cardiology), University of Maryland School of Medicine, Baltimore, Maryland, USA

\begin{abstract}
In this guideline, the International Right Heart Foundation Working Group moves a step forward to develop a common language to describe the development and defects that exemplify the common syndrome of right heart failure. We first propose fundamental definitions of the distinctive components of the right heart circulation and provide consensus on a universal definition of right heart failure. These definitions will form the foundation for describing a uniform nomenclature for right heart circulatory failure with a view to foster collaborative research initiatives and conjoint education in an effort to provide insight into mechanisms of disease unique to the right heart.
\end{abstract}

Keywords: right heart failure, pulmonary hypertension, pulmonary circulation, right ventricle, etiology, physiology, anatomy, classification.

Pulm Circ 2013;3(4):963-967. DOI: 10.1086/674750.

The right ventricle may be said to be made for the sake of transmitting blood through the lungs, not for nourishing them. -William Harvey, Exercitatio Anatomica de Motu Cordis et Sanguinis in Animalibus, 1628

Even as progress in understanding left heart failure has ensued unfettered, the right heart has, for many years, been relegated to a bystander chamber. This downgrade has been accepted for decades under the presumption that the right heart is a conduit structure that is a secondary actor in the interplay of heart failure, with primacy accorded to the left ventricle. Yet the sentinel role of right heart failure in determining functional, end-organ, and clinical outcomes has become the subject of increasing recent inquiry. ${ }^{1}$ We now recognize that the right heart is structurally discrete in its anatomic, electrical, and cellular configuration; develops failure often due to distinct pathobiologic pathways that are separate from the left ventricle; and plays a central role in determining prognosis even as therapeutic success in addressing left ventricular dysfunction is demonstrated. ${ }^{2}$ Uniquely, therapy that influences the left ventricle favorably may not impact the dysfunctional right ventricle, and vice versa. ${ }^{3}$

The right ventricle and left ventricle are anatomically, physiologically, and functionally distinct. The right ventricle is anatomically composed of 3 distinct portions: ${ }^{4}$ the first portion is the inlet, which includes the tricuspid valve, the chordae tendinae, and papillary muscles; the second portion is the trabeculated apical myocardium; and finally, the infundibulum, or conus, constitutes the outlet region. The shape of the right ventricle is complex: it appears triangular from the side and is crescent shaped in cross section. There are 2 layers of the right ventricular myocardium. The fibers of the superficial layer of the right ventricle are arranged circumferentially in a direction that is parallel to the atrioventricular (AV) groove in continuity with the left ventricle. The deep muscle fibers of the right ventricle are longitudinally aligned base to apex (in contrast to the left ventricle, where oblique fibers are found superficially, longitudinal fibers on the endocardium, and circumferential fibers in between). Haddad and colleagues ${ }^{4}$ elegantly described the morphologic differences between the left and right ventricles as follows: (1) a more apically situated hinge point of the septal leaflet of the tricuspid valve relative to the anterior leaflet of the mitral valve, (2) the presence of a moderator band in the right ventricle cavity, (3) more than 2 papillary muscles, (4) a trileaflet atrioventricular valve with septal attachments, (5) predominantly coarse trabeculations, and (6) a

Address correspondence to Mandeep R. Mehra, MD, Medical Director, BWH Heart and Vascular Center, A-3, 75 Francis Street, Boston, MA 02115, USA. E-mail: mmehra@partners.org.

Submitted October 12, 2013; Accepted November 4, 2013; Electronically published February 18, 2014.

(C) 2014 by the Pulmonary Vascular Research Institute. All rights reserved. 2045-8932/2013/0304-0024. \$15.00. 
ventriculoinfundibular fold that separates the tricuspid valve from the pulmonic valve (as opposed to the aortomitral continuity seen on the left). These anatomic structural differences indicate that the designs are intended for distinct purposes on an evolutionary scale. Importantly, they provide insight into the biologic diversity that explains the varied phenotypic reactions to hemodynamic stressors.

Physiologically, the right ventricle is more uniquely sensitive to afterload, demonstrates a trapezoid pressurevolume curve (as opposed to a rectangular pressure-volume loop for the left ventricle), and develops dysfunction by many distinct pathways. ${ }^{5}$ In the setting of an acute increase in pulmonary arterial impedance, as encountered in pulmonary embolism, the right ventricle demonstrates evidence of a severe reduction in stroke volume with a narrow window of pressure increase. ${ }^{6}$ When a left ventricular assist device is placed and ventricular suction applied such that the septum is moved into the left ventricular cavity, one frequently has significant right ventricular dysfunction even with reduced afterload. ${ }^{7}$ Extrinsic compression, as with pericardial constriction or effusion, can impede right ventricular function by compressive dynamics and lead to manifestations of heart failure. ${ }^{8}$ In clinical syndromes of congenital heart disease, one can exhibit the syndrome of right-sided failure due to changes in flow and anatomic defects located within the right ventricle or in anatomic areas preceding blood entry into this chamber. ${ }^{5}$ In chronically raised afterload and pulmonary impedance, the right ventricle demonstrates rather diverse responses, with variable expression of dysfunction over time. Despite these complex attributes, it is clear that development of right-sided dysfunction portends a steep decline in prognosis accompanied by multisystem organ failure, reflected variably in clinical expression as the cardiorenal syndrome, protein-losing enteropathy, and cardiac cachexia. ${ }^{9-11}$

It has become obvious that the complex nature of rightsided heart failure, the diverse pathways and multispecialty involvement among distinct clinicians, such as cardiologists, pulmonologists, congenital heart disease experts, and cardiothoracic surgeons, has enforced the development of varying definitions that are uniformly resident within silos and consequently cover a limited scope and clinical need. To address this impediment to research and innovation in right heart failure, leading experts from around the world in the fields of congenital heart disease, pulmonary vascular disease, congestive heart failure, and cardiothoracic surgery came together under the aegis of the newly founded International Right Heart Foundation with the core mission of bringing this distinguished interdis- ciplinary group of expert physician scientists together to develop an integrative language that effectively captures and describes right heart disease. The development of a common language relevant to scientists and clinicians alike was designed to foster collaborative research initiatives and conjoint education in an effort to provide insight into mechanisms of disease unique to the right heart while advancing patient care.

This group reached united consensus on the basic definitions concerning the right heart and further emphasized that confusion between the commonly used nomenclature of "right ventricular failure" and "right heart failure" must be clarified as follows and not used interchangeably.

Definition 1: distinction between right heart failure and right ventricular failure. Right heart failure represents a disturbance or dysfunction in any of the components that constitute the right heart circulatory system (defined below). Thus, right ventricular failure, in contradistinction, is one component (albeit major) of a pathophysiological entity that can result in right heart circulatory failure.

Definition 2: components of the right heart system. The "right heart circulatory system" is comprised of the systemic veins up to the pulmonary capillaries—at which point deoxygenated blood transitions to oxygenated blood. The right heart system can be classified into systemic and pulmonary circuits. The systemic circuit includes the systemic veins, right atrium, coronary sinus (and cardiac venous drainage), tricuspid valve, right ventricular free wall, right ventricular outflow tract, and pulmonic valve. The pulmonary circuit includes the main pulmonary artery postpulmonic valve and the secondary and tertiary branches of the pulmonary arteries.

The "left heart circulatory system" is comprised of the postpulmonary capillaries to the systemic arteries-at which point oxygenated blood begins to shift to deoxygenated blood. The left heart circulatory system is comprised of the pulmonary veins, left atrium, mitral valve, left ventricle, aortic valve, aorta, and systemic arteries (including the coronary arteries). The pulmonary and systemic capillary beds are shared between the two compartments on the right- and left-sided circulatory system.

Definition 3: what is right heart failure? We define "right heart failure" as a clinical syndrome due to an alteration of structure and/or function of the right heart circulatory system that leads to suboptimal delivery of blood flow (high or low) to the pulmonary circulation and/or elevated venous pressures-at rest or with exercise.

Distinctively, this definition is broad and classifies right heart failure as a syndrome, which may result from anatomic or physiologic aberrations, or both, from a variety 
Table 1. Development of a comprehensive nomenclature of right heart failure: framework, components, and issues

Components, issues

1. Etiology: What is the primary cause?

a. Draw upon existing classification systems for congenital heart disease

b. Advance current descriptions of acquired etiologies (The novel aspect lies in the expanded definition of right heart failure to include precardiac and postpulmonary valvular compartments.)

2. Anatomy: Where is the primary defect?

a. Describe the anatomic constituents of the right heart circulatory system

b. Describe the anatomic defects within the right heart circulatory system (systemic circuit and pulmonary circuit) and left heart circulatory system

3. Physiology: What is the primary aberration?

a. Define the appropriate hemodynamic and nonhemodynamic definitions and assessments of 3 distinct physiologic disturbances in the domains of preload stress, contractile insufficiency, and afterload stress

4. Clinical function: What is the clinical expression in the patient?

a. Functional class components

A. Subjective component

a. Patient's reported symptom (modified NYHA functional class)

b. Assessment of patient's activity profile (sedentary, active, etc.)

c. Quality of life (most appropriate assessment to be determined)

B. Objective component

a. 6MWT (include Borg score, percent predicted value)

b. Pending results and/or clinical situation, proceed with cardiopulmonary stress test (CPEX) (availability, safety, and consistency in interpretation of data presenting as barriers to be further discussed)

i. Assess hemodynamic and/or ventilator response/insufficiency

C. Modifiers to functional assessment (noncardiovascular contributors to symptoms)
a. Body mass index (BMI)
b. Orthopedic limitations
c. Systemic processes

b. How should secondary modifiers (secondary organ function) be incorporated?

i. Renal dysfunction, as measured by GFR?

ii. Hepatic dysfunction, as measured by MELD

(Should these elements be treated under physiologic aberrations or functional aspects?)

Note: NYHA: New York Heart Association; 6MWT: 6-minute walk test; CPEX: cardiopulmonary exercise test; GFR: glomerular filtration rate; MELD: model for end-stage liver disease.

of etiologies that are not restricted to the right ventricle. Importantly, this definition allows for abnormalities to manifest themselves clinically during exercise alone while remaining quiescent during resting conditions. Furthermore, we believe that it is important to allow for a broad definition that encompasses most disorders (ranging from those that always involve the right ventricle to those that may spare the right ventricle yet result in the manifest clinical syndrome such as a pretricuspid lesion). However, we do recognize that clinical exceptions to the rule always exist (e.g., occlusive disease of the inferior vena cava). Thus, it is our contention that this definition, as proposed, may meet the goal of widest incorporation of pathologic substrates and their clinical manifestation that influence clinical expression in the right heart circulatory system.

Toward a comprehensive clinical nomenclature. The next step to build upon these fundamental definitions is to develop a comprehensive nomenclature that can be used by clinicians to accurately standardize and discuss the etioanatomicophysiologic basis that results in clinical manifestations of right heart failure. Current classification systems used to describe disease of the left heart are inadequate 
to capture the unique features of the right heart. Furthermore, epidemiologic studies do not provide guidance by which a classification system distinct to the right heart may be derived. However, past experiences can inform us on developing a cogent structure of a framework to develop this process. One such early attempt to categorize heart disease was developed as a book in 1928 by Paul Dudley White and Merrill M. Myers, whose work was ultimately published as a book in 1928 by the New York Heart Association (NYHA), which classified cardiovascular disease. The most recent version was published in $1994 .{ }^{12}$ Despite its ambiguities and vulnerability to subjective assessment, it remains a hallowed system by which to evaluate clinical cardiovascular disease and is often the endpoint of studies of investigational therapies. ${ }^{13}$ The 5 essential elements of the NYHA classification of cardiovascular disease are etiology, anatomy, physiology, functional status, and objective assessment. Using the same elements and framework developed nearly a century ago, we have put forward a structure for developing the classification system for right heart failure. The salient features of this working structure (and questions that need to be addressed), which will be refined and developed in 2014 by the International Right Heart Foundation Nomenclature Working Group in partnership with sister organizations and societies, are described in Table 1.

In summary, we believe that the International Right Heart Foundation Working Group is poised to develop a common language to describe the development and defects that exemplify this important clinical syndrome. The common fundamental definitions of components of the right heart, distinction between the right heart and subcomponents (e.g., the right ventricle), and consensus on a universal definition of right heart failure represent the first important steps toward scientific progress in this area. We resonate with the declaration of Dr. Eugene Braunwald at our first consensus summit, where he poignantly professed the right heart as a "stepchild no more!"

\section{ACKNOWLEDGMENTS}

We are grateful to the Pulmonary Vascular Research Institute (PVRI) and the International Society for Heart and Lung Transplantation (ISHLT) for providing official representation as we develop the nomenclature. Finally, we thank Dr. Eugene Braunwald for his advice, mentorship, and guidance throughout this process.

We are thankful for the leadership and participation of the International Right Heart Foundation Nomenclature Working Group. The principal consensus definitions were developed at the Right Heart Failure Summit held in Boston on October 12, 2012. In addition to the authors of this article, the following participants attended the first meeting to develop these definitions: William Auger, MD (La Jolla, CA, USA), Maurice Beghetti, MD (Geneva, Switzerland), Raymond L. Benza, MD (Pittsburgh, PA, USA), Hunter Champion, MD, PhD, FACA (Pittsburgh, PA, USA), Paul R. Forfia, MD (Philadelphia, PA, USA), Robert P. Frantz, MD (Rochester, MN, USA), Michael A. Gatzoulis, MD, PhD, FACC, FESC (London, UK), Nicholas S. Hill, MD (Boston, MA, USA), Shaf Keshavjee, MD, MSc, FRCSC, FACS (Toronto, ON, Canada), Robert L. Kormos, MD, FRCS(C), FACS, FAHA (Pittsburgh, PA, USA), Richard A. Krasuski, MD, FACC, FAHA (Cleveland, OH, USA), James E. Lock, MD (Boston, MA, USA), Nicholas W. Morrell, MD (Cambridge, UK), Robert Naeije, MD, PhD (Brussels, Belgium), Alexander R. Opotowsky, MD, MPH (Boston, MA, USA), Andrew N. Redington, MD, FRCP(C), FRCP(UK) (Toronto, ON, Canada), Ivan M. Robbins, MD (Nashville, TN, USA), David M. Systrom, MD (Boston, MA, USA), Patricia A. Uber, BS, PharmD (Baltimore, MD, USA), Jean-Luc Vachiéry, MD (Brussels, Belgium), Anton Vonk Noordegraaf, MD, PhD (Amsterdam, Netherlands), Gary D. Webb, MD (Cincinnati, OH, USA), and Martin Wilkins, MD (London, UK).

Current members of the Right Heart Failure Nomenclature Working Group also include Harm Jan Bogard, MD, PhD (Amsterdam, Netherlands), Ghazwan Butrous, MD, PhD (Canterbury, UK), Phillip C. Camp Jr., MD (Boston, MA, USA), Barbara A. Cockrill, MD (Boston, MA, USA), Steven D. Colan, MD (Boston, MA, USA), Paul Corris (Newcastle, UK), Curt J. Daniels, MD, FACC (Columbus, OH, USA), Teresa DeMarco, MD (San Francisco, CA, USA), Marcelo F. DiCarli, MD (Boston, MA, USA), Harrison Farber (Boston, MA, USA), Sean Gaine, MD (Dublin, Ireland), Tal Geva, MD (Boston, MA, USA), Michael M. Givertz, MD (Boston, MA, USA), Marco Guazzi, MD, PhD (Milan, Italy), Paul M. Hassoun, MD (Baltimore, MD, USA), William E. Hopkins, MD, (Burlington, VT, USA), Marc Humbert, MD, PhD (Clamart, France), Irene Lang, MD (Vienna, Austria), Bradley A. Maron, MD (Boston, MA, USA), Francis X. McGowan Jr., MD, FAAP (Philadelphia, PA, USA), Vallerie V. McLaughlin, MD (Ann Arbor, MI, USA), Mary Mullen, MD (Boston, MA, USA), Francis D. Pagani, MD, PhD (Ann Arbor, MI, USA), Andrew Peacock, MD (Glasgow, UK), Stuart Rich, MD (Chicago, IL, USA), Lewis J. Rubin, MD (San Diego, CA, USA), Olivier Sitbon, MD (Paris, France), Victor F. Tapson, MD (Durham, NC, USA), David Taylor (Cleveland, OH, USA), Gruschen R. Veldtman, MBChB, FRCP (Cincinnati, OH, USA), and Roham T. Zamanian, MD, FCCP (Palo Alto, CA, USA).

The opinions expressed in this article are not necessarily those of the editors or of the ISHLT; the statements do not imply official endorsement as a formal ISHLT document. This article was accepted for dual publication simultaneously in the Journal of Heart and Lung Transplantation and Pulmonary Circulation with editorial consent.

\section{Source of Support: Nil.}

Conflict of Interest: MRM reports consulting for St. Judes, Boston Scientific, Thoratec, Baxter, Johnson and Johnson (Janssen), Medtronic, the National Institutes of Health, and the American Board of Internal Medicine. MHP reports consulting for Actelion Pharmaceuticals, Bayer, Gilead Sciences, and United Therapeutics. MJL reports research funding and trial executive committee 
appointments from Actelion, Myogen, and the National Heart, Lung, and Blood Institute. AL has no conflicts of interest to declare. ABW reports research grant support from United Therapeutics, Gilead Sciences, and Incyte.

\section{REFERENCES}

1. Park MH, Mehra MR. Pulmonary hypertension: the great leveler. J Am Coll Cardiol 2012;59(3):232-234.

2. Redington AN. Physiopathology of right ventricular failure. Semin Thorac Cardiovasc Surg Pediatr Card Surg Annu 2006;9:3-10.

3. Kirkby NS, Hadoke PW, Bagnall AJ, Webb DJ. The endothelin system as a therapeutic target in cardiovascular disease: great expectations or bleak house? Br J Pharmacol 2008;153 (6):1105-1119.

4. Haddad F, Hunt SA, Rosenthal DN, Murphy DJ. Right ventricular function in cardiovascular disease, part I: anatomy, physiology, aging, and functional assessment of the right ventricle. Circulation 2008;117(11):1436-1448.

5. Roche SL, Redington AN. The failing right ventricle in congenital heart disease. Can J Cardiol 2013;29(7):768-778.

6. Lualdi JC, Goldhaber SZ. Right ventricular dysfunction after acute pulmonary embolism: pathophysiologic factors, detec- tion, and therapeutic implications. Am Heart J 1995;130(6): 1276-1282.

7. Cameli M, Lisi M, Righini FM, Focardi M, Lunghetti S, Bernazzali S, Marchetti L, et al. Speckle tracking echocardiography as a new technique to evaluate right ventricular function in patients with left ventricular assist device therapy. J Heart Lung Transplant 2013;32(4):424-430.

8. Dell'Italia LJ. Anatomy and physiology of the right ventricle. Cardiol Clin 2012;30(2):167-187.

9. Tang WH, Mullens W. Cardiorenal syndrome in decompensated heart failure. Heart 2010;96(4):255-260.

10. Johnson JN, Driscoll DJ, O'Leary PW. Protein-losing enteropathy and the Fontan operation. Nutr Clin Pract 2012;27(3): 375-384.

11. Mehra MR. Fat, cachexia, and the right ventricle in heart failure: a web of complicity. J Am Coll Cardiol 2013;62: 1671-1673.

12. Criteria Committee of the New York Heart Association. Nomenclature and criteria for diagnosis of diseases of the heart and great vessels. 9th ed. Boston: Little, Brown, 1994.

13. Hurst JW, Morris DC, Alexander RW. The use of the New York Heart Association's classification of cardiovascular disease as part of the patient's complete problem list. Clin Cardiol 1999;22(6):385-390. 